\title{
III Methodik
}

8 Methodische Überlegungen und Fallmaterial

\subsection{Präzisierung der Fragestellung}

Die in der Einleitung eher allgemein formulierte Fragestellung kann nach der Sichtung themenrelevanter theoretischer Konzepte und empirischer Befunde folgendermaßen präzisiert werden: Wie wird Demenz in den Erlebnisberichten der Betroffenen und ihrer Angehörigen als einzigartiger, höchst subjektiv erlebter Prozess konstruiert? Was bedeutet dies sowohl für die Forschung als auch die praxisorientierte Begleitung demenziell Erkrankter? Was lässt sich aus diesen höchst individuellen Einzelfällen dennoch verallgemeinern? Welche Schlussfolgerungen und Handlungsempfehlungen lassen sich daraus für Betroffene und ihre Angehörigen ableiten, um der Herausforderung Demenz begegnen zu können und sie zu bewältigen?

\subsection{Auswahl des empirischen Korpus}

Die Gewinnung des empirischen Materials erfolgte auf eine etwas ungewöhnliche Weise. Anstatt Betroffene und ihre Angehörigen aufzusuchen und biographisch-narrative Interviews zu führen oder Verhaltensbeobachtungen vorzunehmen, wie Stechl, Höwler, Snyder und Matoff dies taten, oder Fragebögen zu verschicken, wird auf bereits vorhandenes Material zurückgegriffen, das bisher noch nicht in der in Kapitel IV vorgesehenen Weise analysiert aufbereitet und ausgewertet wurde. Ziel ist es, mit Hifle des Fallmaterials zu belegen, dass Verlauf und Bewältigung einer Demenzerkrankung nicht unwesentlich von sozialem Kontext, Persönlichkeitseigenschaften und lebensgeschichtlichen Prägungen beeinflusst werden. Dabei wurde den autobiographischen Erlebnisberichten von McGowin, Bryden und Taylor besondere Bedeutung zugemessen. Auch wenn diese Publikationen aus diversen Gründen (extrem schwache empirische Basis, problematische Selektivität) nicht als repräsentativ betrachtet werden können, erlauben sie doch einen authentischen Blick in die ansonsten verschlossene Welt Demenzkranker.

\subsection{Konkretisierung des methodischen Vorgehens}

Unter den zahlreichen Publikationen wurden die ausgewählt, bei denen themenrelevante Aspekte prägnant formuliert wurden. Zunächst wird das subjektive Krankheitserleben anhand von Porträts im Längsschnitt und dann, in krankheitsbedingte Veränderungsfelder untergliedert, gleichsam im Querschnitt beleuchtet. 